\title{
Two-Step Methods and Bi-Orthogonality
}

\author{
By A. Iserles and S. P. Norsett
}

\begin{abstract}
We study order and zero-stability of two-step methods of Obrechkoff type for ordinary differential equations. A relation between order and properties of $m$ th degree polynomials orthogonal to $x^{\mu_{i}}, 1 \leqslant i \leqslant m$, where $-1<\mu_{1}<\mu_{2}<\cdots<\mu_{m}$, is established. These polynomials are investigated, focusing on their explicit form, Rodrigues-type formulae and loci of their zeros.
\end{abstract}

1. Introduction. There exists a close relationship between certain one-step numerical methods for the ordinary differential equation

$$
y^{\prime}=f(y), \quad y(0)=y_{0} \in \mathbf{R}^{s}
$$

and orthogonal polynomials. This can be demonstrated for linear multi-derivative methods by a construction similar to that in Nørsett [4]. Integrating (1) from $x_{0}$ to $x_{1}=x_{0}+h$, we obtain

$$
y\left(x_{1}\right)-y\left(x_{0}\right)=h \int_{0}^{1} f\left(y\left(x_{0}+t h\right)\right) d t .
$$

Given an arbitrary $m$ th degree polynomial $p$, normalized so that $p^{(m)}(x) \equiv 1$, we proceed by repeated partial integration, obtaining

$$
\begin{aligned}
y\left(x_{1}\right)-y\left(x_{0}\right)= & h \int_{0}^{1} p^{(m)}(1-t) f\left(y\left(x_{0}+t h\right)\right) d t \\
= & -\sum_{i=1}^{m} h^{i}\left\{p^{(m-i)}(0) f^{(i-1)}\left(y\left(x_{1}\right)\right)-p^{(m-i)}(1) f^{(i-1)}\left(y\left(x_{0}\right)\right)\right\} \\
& +h^{m+1} \int_{0}^{1} p(1-t) f^{(m)}\left(y\left(x_{0}+t h\right)\right) d t .
\end{aligned}
$$

Let $Y_{k}$ denote an approximation to $y\left(x_{k}\right), k=0,1$, and let

$$
y^{(i)}=F_{i}(y), \quad i \geqslant 0,
$$

be the equation for the $i$ th derivative that is obtained from (1) by repeated differentiation: $F_{0}(y)=y, F_{1}(y)=f(y), F_{2}(y)=(\partial f(y) / \partial y) f(y)$, etc. The identity (3) gives rise to the $m$ th order method

$$
\sum_{i=0}^{m} h^{i} p^{(m-i)}(0) F_{i}\left(Y_{1}\right)=\sum_{i=0}^{m} h^{i} p^{(m-i)}(1) F_{i}\left(Y_{0}\right)
$$

with the local truncation error

$$
T=h^{m+1} \int_{0}^{1} p(1-t) f^{(m)}\left(y\left(x_{0}+t h\right)\right) d t .
$$

Received February 6, 1985; revised October 23, 1986 and January 9, 1987.

1980 Mathematics Subject Classification (1985 Revision). Primary 65L05, 41A10. 
It follows from (5) that the order can be further increased to $m+s, 1 \leqslant s \leqslant m$, if

$$
\int_{0}^{1} t^{j} p(1-t) d t=0, \quad 0 \leqslant j \leqslant s-1
$$

Hence, the maximal order $2 m$ is attained when $p$ is the $m$ th-degree Legendre polynomial, appropriately shifted,

$$
p(x)=\frac{1}{(2 m) !} \frac{d^{m}}{d x^{m}}\left\{x^{m}(x-1)^{m}\right\} .
$$

Of course, instead of maximally increasing the order, it is possible to seek other properties, e.g., numerical efficiency (Nørsett [4]).

More examples of relations between orthogonality and one-step methods are available in Nørsett [5].

The purpose of the present paper is to extend this set of ideas to two-step methods. To this end, we have to modify the standard concept of orthogonality. This leads to a problem in bi-orthogonality: Find $p_{m}$ in $\pi_{m}$, the set of $m$ th-degree polynomials, which is orthogonal in $[0,1]$ to $x^{\mu_{i}}, 1 \leqslant i \leqslant m$. The $\mu_{i}$ 's are a set of distinct numbers in $(-1, \infty)$-in our application they will be either the even or the odd nonnegative integers.

We demonstrate the existence and uniqueness of such polynomials, obtain explicit expressions and a Rodrigues-type formula and discuss the location of their zeros.

The results are specialized to the problem in hand, namely two-step methods for the equation (1).

2. Two-Step Methods. Given an analytic real function $y$, positive $h$ and arbitrary real $\alpha$, we define the operator $L$ by

$$
L\left[y\left(x_{0}\right) ; h\right]=y\left(x_{1}\right)-(1+\alpha) y\left(x_{0}\right)+\alpha y\left(x_{-1}\right),
$$

where $x_{k}=x_{0}+k h, k=-1,0,1$.

If $y$ is the solution of (1) we obtain

$$
L\left[y\left(x_{0}\right) ; h\right]=h \int_{0}^{1} f\left(y\left(x_{0}+t h\right)\right) d t-\alpha h \int_{0}^{1} f\left(y\left(x_{-1}+t h\right)\right) d t .
$$

In a manner similar to that in Iserles [1], we consider arbitrary $p, q \in \pi_{m}[x]$ such that $p^{(m)}(x) \equiv 1, q^{(m)}(x) \equiv-\alpha$, and repeatedly integrate by parts in (8). This yields

$$
\begin{aligned}
& L\left[y\left(x_{0}\right) ; h\right]= h \int_{0}^{1} p^{(m)}(1-t) f\left(y\left(x_{0}+t h\right)\right) d t \\
&+h \int_{0}^{1} q^{(m)}(1-t) f\left(y\left(x_{-1}+t h\right)\right) d t \\
&=-\sum_{i=1}^{m}\left\{p^{(m-i)}(0) f^{(i-1)}\left(y\left(x_{1}\right)\right)+\left[q^{(m-i)}(0)-p^{(m-i)}(1)\right]\right. \\
&\left.\quad \times f^{(i-1)}\left(y\left(x_{0}\right)\right)-q^{(m-i)}(1) f^{(i-1)}\left(y\left(x_{-1}\right)\right)\right\} h^{i} \\
&+T\left[y\left(x_{0}\right) ; h\right],
\end{aligned}
$$

where the local truncation error operator $T$ has the form

$$
\begin{aligned}
T[y(x) ; h]=h^{m+1} & \left\{\int_{0}^{1} p(1-t) y^{(m+1)}(x+t h) d t\right. \\
& \left.+\int_{0}^{1} q(1-t) y^{(m+1)}(x+(t-1) h) d t\right\} .
\end{aligned}
$$


Hence, the method

$$
\begin{aligned}
\sum_{i=0}^{m} p^{(m-i)}(0) F_{i}\left(Y_{1}\right) h^{i}= & \sum_{i=0}^{m}\left\{p^{(m-i)}(1)-q^{(m-i)}(0)\right\} F_{i}\left(Y_{0}\right) h^{i} \\
& +\sum_{i=0}^{m} q^{(m-i)}(1) F_{i}\left(Y_{-1}\right) h^{i}
\end{aligned}
$$

where $Y_{k}$ denotes an approximation to $y\left(x_{k}\right)$, is of order $p \geqslant m$.

We obtain necessary and sufficient conditions for order $m+r$ by expanding $y^{(m+1)}$ in Taylor series about $x_{0}$,

$$
\int_{0}^{1} t^{j} p(1-t) d t+\int_{0}^{1}(t-1)^{j} q(1-t) d t=0, \quad 0 \leqslant j \leqslant r-1 .
$$

This can be recast as

$$
\int_{0}^{1} t^{j}\left\{p(1-t)+(-1)^{j} q(t)\right\} d t=0, \quad 0 \leqslant j \leqslant r-1 .
$$

If $q \equiv 0$, then we are back to one-step methods, and (11) coincides with (6). In general, alas, the appearance of $(-1)^{j}$ in (11) means that the classical orthogonality analysis is inadequate to provide order conditions on $p$ and $q$.

The situation is relatively simple if either $p$ or $q$ are given. For example, we have

TheOREM 1. Given $p \in \pi_{m}[x], p^{(m)}(x) \equiv 1$, the order of $(10)$ is at least $2 m+1$ if and only if $q$ is of the form

$$
q(x)=\sum_{i=0}^{m} \gamma_{i} P_{i}(x)
$$

where the $P_{i}$ 's are Legendre polynomials, shifted to $[0,1], P_{i}^{(i)}(x) \equiv 1$, and

$$
\gamma_{j}=-\frac{\int_{0}^{1} P_{j}(-t) p(1-t) d t}{\int_{0}^{1} P_{j}^{2}(t) d t}, \quad 0 \leqslant j \leqslant m .
$$

Proof. Order conditions (11) lead to

$$
\int_{0}^{1} P_{l}(-t) p(1-t) d t+\int_{0}^{1} P_{l}(t) q(t) d t=0, \quad 0 \leqslant l \leqslant r-1 .
$$

This follows by multiplying (11) by $\alpha_{j}$, where $P_{l}(t)=\sum_{j=0}^{l} \alpha_{j}(-t)^{j}$, and summing up for $0 \leqslant j \leqslant l$. (Note that Eq. (2.4) in Iserles [1] is incorrect in this respect.) The theorem follows at once from the representation of $q$ and orthogonality of the Legendre polynomials.

To attain zero-stability in (10) it is necessary and sufficient that $-1 \leqslant \alpha<1$, hence, in the framework of Theorem 1 ,

$$
-1 \leqslant \int_{0}^{1} P_{m}(-t) p(1-t) d t / \int_{0}^{1} P_{m}^{2}(t) d t<1 .
$$

Example 2. Let $m=2$, and $p(x)=\gamma^{2}-2 \gamma x+\frac{1}{2} x^{2}$ for a real $\gamma$. Theorem 1 gives

$$
\gamma_{0}=-\gamma^{2}+\gamma-\frac{1}{6}, \quad \gamma_{1}=12 \gamma^{2}-10 \gamma+\frac{3}{2}, \quad \gamma_{2}=-360 \gamma^{2}+240 \gamma-31
$$


This leads to

$$
\begin{aligned}
q(x)= & \left(-180 \gamma^{2}+120 \gamma-\frac{31}{2}\right) x^{2}+\left(192 \gamma^{2}-132 \gamma+17\right) x \\
& +\left(-37 \gamma^{2}+26 \gamma-\frac{1}{2}\right) .
\end{aligned}
$$

The underlying method is of order 5 for all $\gamma \in \mathbf{R}$ and is zero-stable for

$$
\gamma \in\left(\frac{1}{6}, \frac{1}{3}-\frac{\sqrt{5}}{15}\right] \cup\left[\frac{1}{3}+\frac{\sqrt{15}}{15}, \frac{1}{2}\right) .
$$

Order 6 is attained for $\gamma=(9 \pm \sqrt{15}) / 33$, but the corresponding methods are not zero-stable.

In general, there are $2 m+1$ free parameters (including $\alpha$ ) in $p$ and $q$. Hence, it should be possible to attain order $3 m+1$, resulting in $2 m+1$ equations in (11). To this end, we set

$$
U(t):=p(1-t)+q(t), \quad V(t):=p(1-t)-q(t)
$$

and restate (11) for $r=2 m+1$ as

$$
\begin{array}{cl}
\int_{0}^{1} t^{2 j} U(t) d t=0, & 0 \leqslant j \leqslant m \\
\int_{0}^{1} t^{2 j+1} V(t) d t=0, & 0 \leqslant j \leqslant m-1 .
\end{array}
$$

Since (14) represents a nonsingular homogeneous system of linear equations in the coefficients of $U(t)$, it follows that $U(t) \equiv 0$.

Example 3. Given $m=2$, (15) yields

$$
V(t)=\lambda\left(t^{2}-\frac{5}{4} t+\frac{1}{3}\right), \quad \lambda \in \mathbf{R} .
$$

Hence it follows from (13) and $p^{(m)}(x) \equiv 1$ that

$$
p(t)=\frac{1}{2} t^{2}-\frac{3}{8} t+\frac{1}{24}, \quad q(t)=-\frac{1}{2} t^{2}+\frac{5}{8} t-\frac{1}{6} .
$$

Note that $q^{\prime \prime}(t) \equiv-1$, hence the corresponding method is marginally zero-unstable, having a double root at 1 .

The possible absence of zero-stability in the highest-order method motivates interest in methods of order $3 m$, one less than the maximum. In that case our construction is still valid, with the exception that (14) holds for $0 \leqslant j \leqslant m-1$, but not for $j=m$.

3. Bi-Orthogonality. The condition (15) (as well as (14) with $0 \leqslant j \leqslant m-1$ ) are special cases of the general bi-orthogonality problem: Given a set of distinct numbers $\mu_{i}, i=1,2, \ldots$, in $(-1, \infty)$, find $p_{m} \in \pi_{m}[x]$, normalized so that $p_{m}^{(m)}(x)$ $\equiv 1$, such that

$$
\left\langle x^{\mu_{\iota}}, p_{m}(x)\right\rangle:=\int_{0}^{1} x^{\mu_{t}} p_{m}(x) d x=0, \quad 1 \leqslant i \leqslant m .
$$

When $\mu_{i}=i-1$ we obtain the classical shifted Legendre polynomials, whereas the choices $\mu_{i}=2(i-1)$ and $\mu_{i}=2 i-1$ are of interest to the analysis of two-step methods in this paper. 
Definition 4. Given a function $f$ in $C^{1}$ and $\nu \in(-1, \infty)$, we define the differential operator $D_{\nu}$ by

$$
D_{\nu} f(x)=(\nu+1) f(x)+x f^{\prime}(x)=x^{-\nu} \frac{d}{d x}\left\{x^{\nu+1} f(x)\right\} .
$$

LEMMA 5. (a) $D_{\nu}$ maps $\pi_{m}[x]$ onto itself;

(b) $D_{\nu}$ and $D_{\mu}, \nu, \mu \in(-1, \infty)$, commute when acting on a $f \in C^{2}$.

Proof. The assertion (a) is an immediate consequence of the definition of $D_{\nu}$, whereas (b) follows since

$$
D_{\nu} D_{\mu} f(x)=x^{2} f^{\prime \prime}(x)+(3+\mu+\nu) x f^{\prime}(x)+(1+\mu)(1+\nu) f(x)
$$

is symmetric in $\nu$ and $\mu$.

We now define

$$
p_{m}(x)=\frac{1}{m !} \prod_{j=1}^{m}\left(1+m+\mu_{j}\right)^{-1} D_{\mu_{1}} D_{\mu_{2}} \cdots D_{\mu_{m}}\left\{(x-1)^{m}\right\}, \quad m \geqslant 1 .
$$

THEOREM 6. The functions $p_{m}$ defined in (17) belong to $\pi_{m}[x]$, satisfy $p_{m}^{(m)}(x) \equiv 1$, and obey the bi-orthogonality conditions (16).

Proof. It follows at once from Lemma 5 that $p_{m} \in \pi_{m}$ and, by induction, that $p_{m}^{(m)}(x) \equiv 1$. Furthermore, by Lemma $5, D_{\mu_{i}}$ commutes with $D_{\mu_{i}}$, hence

$$
\begin{aligned}
& \left\langle x^{\mu_{k}}, p_{m}(x)\right\rangle=\frac{1}{m !} \prod_{j=1}^{m}\left(1+m+\mu_{j}\right)^{-1} \int_{0}^{1} x^{\mu_{k}} D_{\mu_{k}}\left(\prod_{\substack{i=1 \\
i \neq k}}^{m} D_{\mu_{i}}\right)\left\{(x-1)^{m}\right\} d x \\
& =\frac{1}{m !} \prod_{j=1}^{m}\left(1+m+\mu_{j}\right)^{-1} \int_{0}^{1} \frac{d}{d x}\left\{x^{\mu_{k}+1}\left(\prod_{\substack{i=1 \\
i \neq k}}^{m} D_{\mu_{i}}\right)\left\{(x-1)^{m}\right\}\right\} d x . \\
& =\left.\frac{1}{m !} \prod_{j=1}^{m}\left(1+m+\mu_{j}\right)^{-1} x^{\mu_{k}+1}\left(\prod_{\substack{i=1 \\
i \neq k}}^{m} D_{\mu_{i}}\right)\left\{(x-1)^{m}\right\}\right|_{0} ^{1}=0, \quad 1 \leqslant k \leqslant m,
\end{aligned}
$$

since $\mu_{k}>-1$.

Note that no other normalized polynomials in $\pi_{m}[x]$ may satisfy (16): Writing the orthogonality conditions as equations in coefficients of $p_{m}$ leads to a matrix which is a section of a Cauchy matrix, hence nonsingular.

THEOREM 7. The bi-orthogonal polynomials $p_{m}$ have the explicit form

$$
\begin{aligned}
p_{m}(x)= & \frac{1}{m !} \prod_{j=1}^{m}\left(1+m+\mu_{j}\right)^{-1} \\
& \times \sum_{k=0}^{m}(-1)^{m-k}\left(\begin{array}{c}
m \\
k
\end{array}\right) \prod_{j=1}^{m}\left(k+1+\mu_{j}\right) x^{k}, \quad m \geqslant 1 .
\end{aligned}
$$

Proof. By expanding $(x-1)^{m}$ with the binomial theorem and straightforward differentiation.

Given $\beta \in \mathbf{R}$, we define the generalized factorial function $(\beta, \mu)_{m}$ by

$$
(\beta, \mu)_{m}:=\prod_{j=1}^{m}\left(\beta+\mu_{j}\right)
$$


Note that if $\mu_{j}=j-1$ this reduces to the classical factorial function, $(\beta, \mu)_{m}=$ $(\beta)_{m}$.

In the present notation,

$$
p_{m}(x)=\frac{1}{m !} \frac{1}{(m+1, \mu)_{m}} \sum_{k=0}^{m}(-1)^{m-k}\left(\begin{array}{c}
m \\
k
\end{array}\right)(k+1, \mu)_{m} x^{k}, \quad m \geqslant 1 .
$$

Example 8. The case $m=2, \mu_{1}=1, \mu_{2}=3$ gives

$$
p_{2}(x)=\frac{1}{2} x^{2}-\frac{5}{8} x+\frac{1}{6} \text {. }
$$

This can be identified with the polynomial $V$ from Example 3.

It is well known that the classical orthogonal polynomials possess all their zeros in the support of the measure, that the zeros are simple and that zeros of consecutive polynomials interlace. The following theorem shows that all this remains valid for our bi-orthogonal polynomials.

THEOREM 9. Each $p_{m}$ possesses $m$ distinct zeros in $(0,1)$. If the $\mu_{j}$ 's form a monotonically increasing sequence, then the zeros of $p_{m-1}$ and $p_{m}$ interlace.

Proof. The first part of the theorem follows at once from the Rodriguestype formula (17) by repeated application of Rolle's theorem to the functions $x^{\mu_{m}+1}(x-1)^{m}, x^{\mu_{m-1}+1} D_{\mu_{m}}(x-1)^{m}$ and so on.

Moreover, given

$$
\varphi_{m}(x):=\frac{m+1+\mu_{m}}{m !} D_{\mu_{1}} D_{\mu_{2}} \cdots D_{\mu_{m-1}}\left\{(x-1)^{m}\right\}
$$

it follows from (17) that

$$
\begin{aligned}
& (m, \mu)_{m-1} p_{m-1}(x)+\varphi_{m}(x) \\
& \quad=\frac{1}{m !} D_{\mu_{1}} \cdots D_{\mu_{m-1}}\left\{m(x-1)^{m-1}+\left(m+1+\mu_{m}\right)(x-1)^{m}\right\} \\
& =\frac{1}{m !} D_{\mu_{1}} \cdots D_{\mu_{m}}\left\{(x-1)^{m}\right\}=(m+1, \mu)_{m} p_{m}(x), \quad m \geqslant 2 .
\end{aligned}
$$

Hence,

$$
\varphi_{m}(x)=(m+1, \mu)_{m} p_{m}(x)-(m, \mu)_{m-1} p_{m-1}(x) .
$$

Repeated application of the Rolle theorem to $\varphi_{m}$, as defined in (18), shows that $\varphi_{m}$ has $m-1$ distinct zeros in $(0,1)$, in addition to a zero at 1 .

It is straightforward to obtain from (18) the recurrence relation

$$
x \varphi_{m}^{\prime}(x)=m(m+1, \mu)_{m} p_{m}(x)+\left(1+\mu_{m}\right)(m, \mu)_{m-1} p_{m-1}(x) .
$$

Let us suppose that $\hat{x} \in(0,1]$ exists such that $p_{m}(\hat{x})=p_{m-1}(\hat{x})=0$. Then it follows from (19) and (20) that

$$
\varphi_{m}(\hat{x})=\varphi_{m}^{\prime}(\hat{x})=0,
$$

which is impossible. We conclude that zeros of $p_{m-1}$ and $p_{m}$ are distinct.

It is known from the classical theory of orthogonal polynomials that interlacing occurs for the choice $\mu_{j}=j-1, j \geqslant 1$. Hence, if the sequence $\left\{\mu_{j}\right\}$ is monotonically increasing, a continuity argument completes the proof that the zeros of $p_{m-1}$ and $p_{m}$ interlace, since no zero of $p_{m-1}$ may cross a zero of $p_{m}$ when the sequence 
$\{0,1, \ldots, m-1\}$ is deformed into $\left\{\mu_{1}, \ldots, \mu_{m}\right\}$ in a continuous manner and so that all components remain distinct throughout this process.

Note that if $\mu_{j}=\beta+j-1, j \geqslant 1$, for some $\beta>-1$, then the bi-orthogonal polynomials $p_{m}$ can be identified with the (shifted) Jacobi polynomials $P_{m}^{(0, \beta)}$. This identification with standard orthogonal polynomials provides much information on the loci of zeros, as well as recurrence relations, a Christoffel-Darboux formula, etc. Hence, it is of interest to check whether, for other choices of $\left\{\mu_{j}\right\}$, the $p_{m}$ 's can be identified with orthogonal polynomials with positive weight functions (acting, possibly, on a different real interval). Our next result demonstrates that this is not the case.

THEOREM 10. Let $p_{m}$ satisfy (16) and suppose that

$$
\int_{c}^{d} x^{k} p_{m}(x) d \alpha(x)=0, \quad 0 \leqslant k \leqslant m-1
$$

where $\alpha$ is a function of bounded variation and $[c, d]$ is a real interval. Then, necessarily, $c=0, d=1, d \alpha(x)=x^{\beta} d x$ and $\mu_{j}=\beta+j-1$ for some $\beta>-1$.

Proof. Let us suppose that (21) is satisfied. Then, as is well known, the $p_{m}$ 's satisfy the three-term recurrence relation

$$
x p_{m}(x)=a_{m} p_{m+1}(x)+b_{m} p_{m}(x)+c_{m} p_{m-1}(x), \quad m \geqslant 1,
$$

where $a_{m}, c_{m} \neq 0$. We multiply (22) by $x^{\mu}$ for $j$ in $\{1, \ldots, m-1\}$ and integrate between 0 and 1. By bi-orthogonality, the right-hand side vanishes, hence

$$
\int_{0}^{1} x^{1+\mu_{j}} p_{m}(x) d x=0, \quad 1 \leqslant j \leqslant m-1
$$

Let

$$
H_{m}(\mu):=(1+\mu)_{m} \int_{0}^{1} x^{\mu} p_{m}(x) d x, \quad \mu>-1 .
$$

$H_{m}$ is a polynomial in $\pi_{m}[\mu]$ that, by bi-orthogonality, vanishes for $\mu=\mu_{j}, 1 \leqslant j \leqslant m$. Hence,

$$
H_{m}(\mu)=C_{m} \prod_{j=1}^{m}\left(\mu-\mu_{j}\right), \quad C_{m} \neq 0 .
$$

Since, by (23), $H_{m}\left(1+\mu_{j}\right)=0$, it follows that $1+\mu_{j} \in\left\{\mu_{1}, \ldots, \mu_{m}\right\}, 1 \leqslant j \leqslant m-$ 1. Let us assume, without loss of generality, that $\mu_{1}<\mu_{2}<\cdots<\mu_{m-1}$. Then we obtain $\mu_{j+1}=1+\mu_{j}, 1 \leqslant j \leqslant m-1$, hence $\mu_{j}=\beta+j-1$ for some $\beta \in \mathbf{R}$. Since $\beta>-1$ is necessary for integrability, the theorem is proved.

4. Two-Step Approximations to $\exp (z)$. The application of the method (4) to the test equation $y^{\prime}=\lambda y$ yields

$$
Y_{1}=R(z) Y_{0}, \quad z=\lambda h
$$

where

$$
R(z)=\sum_{i=0}^{m} p^{(m-i)}(1) z^{i} / \sum_{i=0}^{m} p^{(m-i)}(0) z^{i}:=Q_{0}(z) / Q_{1}(z)
$$

is an approximation to $\exp (z)$ of order at least $m$ for every $p \in \pi_{m}$. Furthermore, if $p$ is the (scaled and shifted) Legendre polynomial, then the order is $2 \mathrm{~m}$. 
The order relation can be rewritten as

$$
Q_{1}(z) e^{z}-Q_{0}(z)=\mathcal{O}\left(z^{p+1}\right), \quad m \leqslant p \leqslant 2 m
$$

motivating an extension of the present framework to two-step approximations

$$
Q_{1}(z) e^{z}-Q_{0}(z)-Q_{-1}(z) e^{-z}=\mathcal{O}\left(z^{p+1}\right), \quad m \leqslant p \leqslant 3 m+1 .
$$

Shafer [6] has already considered general quadratic approximations of order $p=3 \mathrm{~m}$ +1 to a sufficiently smooth function $f$,

$$
Q_{1}(z) f^{2}(z)-Q_{0}(z) f(z)-Q_{-1}(z)=\mathcal{O}\left(z^{3 m+2}\right), \quad Q_{-1}, Q_{0}, Q_{1} \in \pi_{m}[z] .
$$

He obtained expressions of closed form for the coefficients in (24) with $p=3 m+1$. Likewise, by our analysis in Section 3 it is apparent that

$$
\begin{aligned}
Q_{1}(z) & =\sum_{i=0}^{m} p^{(m-i)}(0) z^{i} \\
Q_{0}(z) & =\sum_{i=0}^{m}\left(p^{(m-i)}(1)-q^{(m-i)}(0)\right) z^{i} \\
Q_{-1}(z) & =\sum_{i=0}^{m} q^{(m-i)}(1) z^{i}
\end{aligned}
$$

where $p(1-x) \equiv-q(x) \equiv(-1)^{m} p_{m}(x)$ with $\mu_{j}=2 j-1,1 \leqslant j \leqslant m$.

LEMMA 11. The maximal-order method is zero-stable if and only if $m$ is odd.

Proof. The assertion follows immediately from $\alpha=-q^{(m)}(x)=(-1)^{m} p_{m}^{(m)}(x)=$ $(-1)^{m}$, since $-1 \leqslant \alpha<1$ is necessary and sufficient for zero-stability.

Hence, since no zero-stability occurs for even $m$ and only marginal zero-stability for odd $m$, it makes sense to sacrifice one degree of freedom in order to enhance stability, thus demanding order $3 m$. The natural choice is $\alpha=0$, leading to Adams-type schemes.

To this end, we consider (14) and (15) (both with $0 \leqslant j \leqslant m-1$ ). By the analysis of Section 3,

$$
\begin{aligned}
& U(x)=C_{1} \frac{(-1)^{m}}{m !} \prod_{j=1}^{m}(m+2 j-1)^{-1} \sum_{k=0}^{m}(-1)^{k}\left(\begin{array}{c}
m \\
k
\end{array}\right) \prod_{j=1}^{m}(k+2 j-1) x^{k}, \\
& V(x)=C_{2} \frac{(-1)^{m}}{m !} \prod_{j=1}^{m}(m+2 j)^{-1} \sum_{k=0}^{m}(-1)^{k}\left(\begin{array}{c}
m \\
k
\end{array}\right) \prod_{j=1}^{m}(k+2 j) x^{k} .
\end{aligned}
$$

The constants $C_{1}$ and $C_{2}$ are determined from (13) by the conditions $p^{(m)}(x) \equiv 1$, $q^{(m)}(x) \equiv 0$, which give $C_{1}=C_{2}=(-1)^{m}$. Hence,

$$
p(x)=\frac{1}{2}(U(1-x)+V(1-x)), \quad q(x)=\frac{1}{2}(U(x)-V(x)),
$$

resulting in an order- $3 m$ zero-stable scheme.

Another interesting choice of $p$ and $q$ in (10) leads to singly-implicit approximations, with a single pole of multiplicity $m$, that were discussed by Iserles [1]. They follow from

$$
p(x)=(-1)^{m} \gamma^{m} L_{m}(x / \gamma),
$$

where $L_{m}$ is the $m$ th Laguerre polynomial

$$
L_{m}(x)=\sum_{i=0}^{m} \frac{(-1)^{i}}{i !}\left(\begin{array}{c}
m \\
i
\end{array}\right) x^{i},
$$


and possess order $2 m$. It has been proved in Iserles [1] that for $1 \leqslant m \leqslant 3$ there exist $\gamma>0$ that yield $A$-acceptability.

Yet another option is to choose

$$
q(x)=(-1)^{m} p(1-x),
$$

obtaining a symmetric method, $Q_{-1}(z) \equiv Q_{1}(z)$.

We do not address here the question of stability for stiff systems. Since attention has been focused on methods of order $p \geqslant 2 m+1, A$-stability is out of the question (Wanner et al. [7]). (The methods in Iserles [1] are an exception, since there, $p=2 m$.) Hence, less severe stability requirements, e.g., $A(\alpha)$-stability and stiffstability, need to be analyzed in this context.

5. Extensions of Bi-Orthogonality. The bi-orthogonal polynomials $p_{m}$ with $\mu_{j}=$ $2 j-1$ can be related to generalized hypergeometric functions via the representation

$$
\begin{aligned}
p_{m}(x)= & \frac{(-1)^{m}}{((m+2) / 2)_{m}{ }_{3}} F_{2}\left[\begin{array}{c}
-\frac{1}{2} m,-\frac{1}{2}(m-1), m+1 ; \\
\frac{1}{2}, 1 ;
\end{array}\right] \\
& +\frac{(-1)^{m+1}(3 / 2)_{m}}{(m-1) !((m+2) / 2)_{m}} x_{3} F_{2}\left[\begin{array}{c}
-\frac{1}{2}(m-1),-\frac{1}{2}(m-2), m+\frac{3}{2} ; \\
\frac{3}{2}, \frac{3}{2} ;
\end{array}\right] .
\end{aligned}
$$

This follows by splitting $p_{m}$ from Theorem 7 , with the above-mentioned parameters, into even and odd polynomials and by using the factorial identities

$$
\begin{gathered}
(\alpha)_{2 k}=4^{k}\left(\frac{\alpha}{2}\right)_{k}\left(\frac{\alpha+1}{2}\right)_{k}, \quad\left(\begin{array}{c}
m \\
k
\end{array}\right)=(-1)^{k} \frac{(-m)_{k}}{(1)_{k}}, \\
(1+\alpha+k)_{m}=\frac{(1+\alpha)_{m}(1+m+\alpha)_{k}}{(1+\alpha)_{k}} .
\end{gathered}
$$

Another, easily obtainable, hypergeometric representation, which is valid for every choice of $\mu_{1}, \mu_{2}, \ldots$, is

$$
p_{m}(x)=\frac{1}{m !}{ }_{m+1} F_{m}\left[\begin{array}{c}
-m, 2+\mu_{1}, 2+\mu_{2}, \ldots, 2+\mu_{m} ; \\
1+\mu_{1}, 1+\mu_{2}, \ldots, 1+\mu_{m} ;
\end{array}\right] .
$$

The concept of bi-orthogonality can be extended in a natural manner:

I. Given a real function $\alpha(x)$ of bounded variation with the support $(a, b)$, a nonnegative function $\omega(x, \mu), x \in(a, b), \mu \in \Omega$, which is not identically zero in $(a, b)$ for every $\mu \in \Omega$, and a sequence $\left\{\mu_{j}\right\}_{j=1}^{\infty} \in \Omega$, find monic $p_{m} \in \pi_{m}[x]$ such that

$$
\int_{a}^{b} p_{m}(x) \omega\left(x, \mu_{k}\right) d \alpha(x)=0, \quad 1 \leqslant k \leqslant m
$$

This problem has been explored in Iserles and Nørsett [2] and has been further discussed in [3]. 
II. Given $\mu_{1}, \mu_{2}, \ldots \in(-1, \infty)$ and $\nu_{1}, \nu_{2}, \ldots \in(0, \infty)$, find $p_{m}$ in $\operatorname{span}\left\{x^{\nu_{1}}, \ldots, x^{\nu_{m+1}}\right\}$ such that

$$
\int_{0}^{1} p_{m}(x) x^{\mu_{k}} d x=0, \quad 1 \leqslant k \leqslant m .
$$

This problem will be the subject of a forthcoming paper.

Many other interesting problems are implicit in the construction of bi-orthogonal polynomials: quadrature, interpolation, representation, $L_{p}$ approximation, etc. The present paper makes only preliminary inroads into this new type of orthogonality.

Department of Applied Mathematics and Theoretical Physics

University of Cambridge

Cambridge CB3 2EW, England

Department of Numerical Mathematics

Norwegian Institute of Technology

N-7034 Trondheim-NTH, Norway

1. A. ISERLES, “Two-step numerical methods for parabolic differential equations," BIT, v. 21, 1981, pp. $80-96$.

2. A. Iserles \& S. P. Nørsetr, "Bi-orthogonal polynomials," in Orthogonal Polynomials and Their Applications (A. Draux, A. Magnus and P. Maroni, eds.), Lecture Notes in Math., vol. 1171, SpringerVerlag, Berlin, 1985, pp. 92-100.

3. A. Iserles \& S. P. Nørsetr, On the Theory of Bi-Orthogonal Polynomials, Tech. Rep. NA1, DAMTP, University of Cambridge, 1986.

4. S. P. NøRSETT, "One-step methods of Hermite type for numerical integration of stiff systems," $B I T$, v. 14,1974 , pp. $63-77$.

5. S. P. NøRSETT, "Splines and collocation for ordinary initial value problems," in Approximation Theory and Spline Functions (S. P. Singh, J. H. W. Burry and B. Watson, eds.), NATO ASI Series, Vol. C136, 1983, pp. 397-417.

6. R. E. Shafer, “On quadratic approximation,” SIAM J. Numer. Anal., v. 7, 1974, pp. 447-460.

7. G. Wanner, E. Hairer \& S. P. NørSeTt, “Order stars and stability theorems,” BIT, v. 18, 1978, pp. $475-489$. 